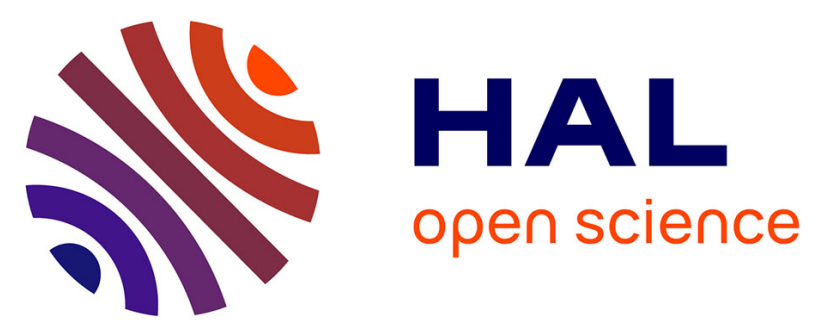

\title{
Impact of the Charging Demand of Electric Vehicles on Distribution Grids: a Comparison Between Autonomous and Non-Autonomous Driving
}

\author{
Fabrizio Sossan, Biswarup Mukherjee, Zechun Hu
}

\section{- To cite this version:}

Fabrizio Sossan, Biswarup Mukherjee, Zechun Hu. Impact of the Charging Demand of Electric Vehicles on Distribution Grids: a Comparison Between Autonomous and Non-Autonomous Driving. 15th International Conference on Ecological Vehicles and Renewable Energies (EVER 2020), Sep 2020, Monte-Carlo, Monaco. pp.1-6, 10.1109/EVER48776.2020.9243122 . hal-03091698

\section{HAL Id: hal-03091698 \\ https://hal.science/hal-03091698}

Submitted on 31 Dec 2020

HAL is a multi-disciplinary open access archive for the deposit and dissemination of scientific research documents, whether they are published or not. The documents may come from teaching and research institutions in France or abroad, or from public or private research centers.
L'archive ouverte pluridisciplinaire HAL, est destinée au dépôt et à la diffusion de documents scientifiques de niveau recherche, publiés ou non, émanant des établissements d'enseignement et de recherche français ou étrangers, des laboratoires publics ou privés. 


\section{Impact of the Charging Demand of Electric Vehicles on Distribution Grids: a Comparison Between Autonomous and Non-Autonomous Driving}

\author{
Fabrizio Sossan and Biswarup Mukherjee \\ PERSEE \\ Mines ParisTech - PSL University \\ Sophia Antipolis, France \\ fabrizio.sossan@mines-paristech.fr
}

\author{
Zechun $\mathrm{Hu}$ \\ Department of Electrical Engineering \\ Tsinghua University \\ Beijing, China \\ zechhu@tsinghua.edu.cn
}

\begin{abstract}
Charging many electric vehicles (EVs) might cause violations of cable ampacities, statutory voltage limits, and substation transformer ratings in power distribution grids. Besides affecting mobility patterns, autonomous driving will open new perspectives in terms of interactions with the power grid. This paper explores the potential of autonomous EVs of reducing grid congestions thanks to the possibility of reaching the most suitable recharging locations autonomously. We first develop an algorithm for the coordinated charging of non-autonomous EVs accounting for grid constraints. We then augment its formulation by modeling the charging locations as decision variables of the problem, adopting an efficient linear mixed-integer program based on a linearized grid model and McCormick (exact) relaxations to handle some bi-linear terms appearing in the formulation. Considering the CIGRE' benchmark system for $L V$ residential grids, we compare non-autonomous versus autonomous EVs and show that the additional degree of freedom coming from autonomous driving achieves reducing grid congestions.

Index Terms-Smart charging; Autonomous Driving; Distribution Networks
\end{abstract}

\section{INTRODUCTION}

A challenge associated with the wide-spread adoption of electric vehicles (EVs) is managing the simultaneous charging demand of large populations of vehicles that might determine violations of constraints in distribution networks and require grid reinforcements [1], [2].

Smart charging refers to distributing the charge of EVs over a longer time span and is known to reduce the peak demand and its effects, see e.g. [3]. The problem of scheduling the charge of EVs to respect grid voltage levels and avoiding (power) congestions was addressed, e.g., in [4], [5], where authors propose an iterative procedure to determine progressively tighter power constraints for the EVs when grid congestions appear, accounting also for electricity wholesale market prices. The work in [6] proposes a market framework for EVs fleet operators, distribution system operators (DSOs), and load balance responsible for satisfying the charging demand of EVs, respecting grid constraints, and providing regulating power to the system. The work in [7] proposes a centralized scheduler based on a non-convex optimal power flow problem. The work in [8] proposes grid-aware transactive energy management for an EVs fleet.

In the meanwhile, technologies for autonomous driving are evolving. Autonomous driving is anticipated to disrupt the way we intend transportation and mobility, with implications ranging from ownership schemes for vehicles (favoring carsharing and ride-hailing options) to mobility demand (that might increase, fostered by more accessible transportation), see ,e.g., [9]. When considering large-scale integration of EVs in power grids, autonomous driving will allow vehicles to independently select the most suitable charging locations (e.g., one near a renewable power plant or energy storage facility), offering a new lever to avoid grid congestions. If future mobility is autonomous, grid reinforcements and technological developments planned today for non-autonomous EVs might become obsolete.

The problem of integrating autonomous electric vehicles (AEVs) in distribution grids is not yet entirely explored in the existing literature. The charge scheduling problem for AEVs was recently addressed in [10] considering the minimization of the waiting times and the electricity costs, neglecting, however, power grid constraints. The problem of planning the charging infrastructure of AEVs was addressed in [11], [12] considering mobility patterns of ride-hailing, without considering, however, distribution grids.

The question that we tackle in this paper is: can autonomous driving reduce the impact of the charging demand of EVs on power distribution grids? To reply, we consider a smart charging scheme for non-autonomous EVs and augment it so that the charging locations of EVs also become a decision variable of the problem. In this way, AEVs can autonomously select the most suitable charging locations in the grid to satisfy their charging demand while respecting grid constraints. We compare these two cases under the same conditions (i.e., the same number of vehicles, and same charging objectives) and accounting for the additional charging demand incurred by $\mathrm{AEV}$ s to drive to the most convenient charger.

The rest of this paper is organized as follows. Section II 
explains the models. Section III presents the smart charging algorithm for non-autonomous and autonomous EVs. Section IV presents the case study and the results. Finally, Section V concludes the papers and draws the main conclusions.

\section{Models of EVs And GRID Constraints}

\section{A. EV battery charging model}

Let $P_{t v}^{(\mathrm{EV})}$ be the charging demand of vehicle $v$ at time $t$. As we consider smart charging, we say that $P_{t v}^{(\mathrm{EV})}$ is non-negative. The SOC of vehicle $v=1, \ldots, V$ is:

$$
\begin{aligned}
\operatorname{SOC}_{t v}\left(P_{t v}^{(\mathrm{EV})}\right) & =\operatorname{SOC}_{t-1 v}\left(P_{(t-1) v}^{(\mathrm{EV})}\right)+\eta \frac{1}{E_{v}} P_{t v}^{(\mathrm{EV})} T_{s}, \\
\text { with } P_{t v} & \geq 0
\end{aligned}
$$

where $\eta$ is the (constant) charging efficiency, $E_{v}$ is the battery energy capacity, and $T_{s}$ the sample time in hours.

Even if at the current stage we do not consider V2G (where EVs can also inject power into the grid and $P_{t v}$ can be negative), the SOC evolution in (1) can be easily augmented to accommodate negative charging demand. A way to do so is splitting the battery power into mutually exclusive positive and negative parts weighted by the efficiency and its inverse, respectively, as commonly done in the literature, see e.g., [13], [14]. Alternatively, when the problem is coupled with grid constraints, one can incorporate the battery losses in an equivalent series resistance with a new virtual node in the grid topology, as proposed in [15], [16], allowing to retain linear constraints when adopting linearized grid models.

1) Charging location of EVs: Let $n=1, \ldots, N$ denote the grid node index. We encode the EVs' charging locations with $N \times V$ binary variables $b_{n v}$ that are 1's if vehicle $v$ charges at node $n$, 0 's otherwise. For non-autonomous EVs, $b_{n v}$ 's are set a-priori (being the charging locations matching with the parking sites) and are an input to the problem. For autonomous EVs, they are free variables of the problem.

2) Nodal injections: The active and reactive power demand at each node of the grid is modelled as the sum of the net demand (i.e., conventional power demand minus distributed stochastic generation, if available) and the aggregated charging demand of all the EVs connected to that node. For time $t$ and node $n$, it is:

$$
P_{t n}\left(P_{t 1}, \ldots, P_{t V}, b_{n 1}, \ldots, b_{n V}\right)=P_{t n}^{(\mathrm{net})}+\sum_{v=1}^{V} b_{n v} P_{t v}^{(\mathrm{EV})} \text {. }
$$

The net demand $P_{t n}^{(\text {net })}$ is an input of the problem and is from point predictions. The binary variable $b_{n v}$ associates the charging demand of vehicle $v$ to node $b$ when active. For convenience in the following formulation, we collect all the variables of (2) in the vectors:

$$
\begin{aligned}
\boldsymbol{P}_{t}^{(\mathrm{EV})} & =\left[P_{t 1}^{(\mathrm{EV})}, \ldots, P_{t V}^{(\mathrm{EV})}\right] \\
\boldsymbol{b}_{n} & =\left[b_{n 1}, \ldots, b_{n V}\right] .
\end{aligned}
$$

We assume voltage-independent power demand. As far as the reactive power is concerned, the reactive power of the net demand is also from point predictions (i.e., derived from the active power demand by assuming a certain power factor), whereas the one of EVs is zero as we consider that EV chargers operate at a unitary power factor. The inclusion of the reactive power as a control variable, that might have an impact on voltage profiles in lines with a nonnegligible reactance of the longitudinal parameters (that is not our case study), can be accommodated easily in the formulation and will be considered in future works.

3) Load flow equations: We use load flow equations to model the magnitudes of the nodal voltages and line currents as a function of the active and reactive nodal injections, grid admittance matrix Y (built from information on the grid topology and on cables parameters), and voltage magnitude at the grid connection point (GCP) $v_{0}$. Let $v_{t n}$ and $i_{t l}$ be the voltage magnitude at node $n$ and current magnitude in line $l$, respectively, at time interval $t$. We denote the load flow equations by

$$
\begin{aligned}
& v_{t n}\left(\boldsymbol{P}_{t}^{(\mathrm{EV})}, \boldsymbol{b}_{n}\right)=f_{n}\left(P_{1}(\cdot), \ldots, P_{N}(\cdot), v_{0}, Y\right) \\
& i_{t l}\left(\boldsymbol{P}_{t}^{(\mathrm{EV})}, \boldsymbol{b}_{n}\right)=h_{l}\left(P_{1}(\cdot), \ldots, P_{N}(\cdot), v_{0}, Y\right),
\end{aligned}
$$

where we have highlighted the dependencies on the EVs' charging demand and charging locations, which will be the decision variables in the scheduling problem. The complex power absorbed from the (single) GCP is denoted by:

$$
S_{t}\left(\boldsymbol{P}_{t}^{(\mathrm{EV})}, \boldsymbol{b}_{n}\right)=g\left(P_{1}(\cdot), \ldots, P_{N}(\cdot), v_{0}, Y\right) .
$$

Functions $f_{1}, \ldots, f_{N}, h_{1}, \ldots, h_{L}$, and $g$ are notoriously nonlinear and, when used in optimization problems, lead to nonconvexities and low tractability. We resort to sensitivity coefficients using the method described in [17] to linearize load flow equations and obtain an approximate solution with a more efficient problem formulation. At this stage, we consider balanced grids, so we carry out a single-phase equivalent load flow. The extension to three-phase systems with linearized grid models is straightforward and does not impact on the formulation, which remains applicable to the more general case.

\section{SCHEDULING THE CHARGE OF NON-AUTONOMOUS AND AUTONOMOUS EVS: PROBLEM FORMULATION}

The problem determines the charging schedule of all vehicles $v=1, \ldots, V$ over the time horizon $t=1, \ldots, T$ to achieve a target SOC while respecting all grid constraints. The target state-of-charge of each vehicle $v$ is denoted by $\mathrm{SOC}_{v}^{*}$. It should be designed to meet the future driving demand based on driver's input or forecasted based on historical values. In this paper, it is assumed given. By resorting to the models introduced in the previous section, we now formulate the scheduling problems, for traditional EVs first, and for autonomous EVs later.

\section{A. Scheduling the charge of non-autonomous EVs}

The charging locations for traditional EVs are fully specified by their parking locations. Specifying the charging locations in 
the modeling framework introduced above entails setting the binary variables $b_{n v}$ to some known values before computing the schedule. In case the scheduling problem needs to be carried out with a certain notice before operations (e.g., to coordinate with intra-day electricity or ancillary service markets), the parking and charging locations can be assumed from point predictions or probabilistic forecasts (for deterministic or stochastic decision, respectively). In certain cases, we expect point predictions to be fairly accurate as, for instance, when EVs are charged overnight at the owners' households. We propose a deterministic formulation where the charging locations are known from point predictions and encoded in the variables $b_{n v}^{*}$. The problem consists in determining the schedule of the charging demand of all vehicles conditioned to knowing charging locations $b_{n v}^{*}$ such that grid constraints are respected. Since, at the current stage, we focus on evaluating distribution grids constraints, we do not consider an electricity price signal from an electricity market. However, the proposed formulation can easily accommodate this by augmenting the cost function. As the cost of charging is a linear function of the charging power, this will not impact on the tractability properties of the problem. Given with $b_{n v}^{*}$, the charge schedule is determined by the following optimization problem:

$$
\underset{P_{11}^{(\mathrm{EV})}, \ldots, P_{T V}^{(\mathrm{EV})} \in \mathbb{R}_{+}}{\arg \min }\left\{\sum_{t=1}^{T} \sum_{v=1}^{V}\left(\mathrm{SOC}_{t v}\left(P_{t v}^{\mathrm{EV}}\right)-\mathrm{SOC}_{v}^{*}\right)^{2}\right\}
$$

subject to EVs' SOC models and power rating limits $\bar{P}_{v}^{(E V)}$ of the chargers ${ }^{1}$ for $t=1, \ldots, T$ and $v=1, \ldots, V$

$$
\begin{aligned}
& \operatorname{SOC}_{t v}\left(P_{t v}^{(\mathrm{EV})}\right)=\operatorname{SOC}_{t-1 v}\left(P_{t v}^{(\mathrm{EV})}\right)+\eta \frac{1}{E_{v}} P_{t v}^{(\mathrm{EV})} T_{s} \\
& 0 \leq \mathrm{SOC}_{t v} \leq 1 \\
& P_{t v}^{(\mathrm{EV})} \leq \bar{P}_{v}^{(\mathrm{EV})}
\end{aligned}
$$

nodal injections model for all $t, v$, and $n=1, \ldots, N$

$$
P_{t n}\left(\boldsymbol{P}_{t}^{(\mathrm{EV})}\right)=P_{t n}^{(\mathrm{net})}+\sum_{v=1}^{V} b_{n v}^{*} P_{t v}^{(\mathrm{EV})}
$$

load flow equations for nodal voltages, lines currents, and power flow at the GCP for all $t$

$$
\begin{array}{ll}
v_{t n}\left(\boldsymbol{P}_{t}^{(\mathrm{EV})}\right)=f_{n}\left(\boldsymbol{P}_{t}^{(\mathrm{EV})}, \boldsymbol{b}_{n}^{*}\right) & n=1, \ldots, N \\
i_{t l}\left(\boldsymbol{P}_{t}^{(\mathrm{EV})}\right)=h_{l}\left(\boldsymbol{P}_{t}^{(\mathrm{EV})}, \boldsymbol{b}_{n}^{*}\right) & l=1, \ldots, L \\
S_{t}\left(\boldsymbol{P}_{t}^{(\mathrm{EV})}\right)=g\left(\boldsymbol{P}_{t}^{(\mathrm{EV})}, \boldsymbol{b}_{n}^{*}\right) &
\end{array}
$$

which should observe, respectively, statutory voltage levels $\underline{v}, \bar{v}$, cables' ampacities $\bar{i}_{l}, l=1, \ldots, L$, and the apparent power rating $\bar{S}$ at the substation transformer for all $t$

$$
\begin{array}{ll}
\underline{v} \leq v_{t n}(\cdot) \leq \bar{v} & n=1, \ldots, N \\
i_{t l}(\cdot) \leq \overline{i_{t l}} & l=1, \ldots, L \\
S_{t}(\cdot)<\bar{S} . &
\end{array}
$$

\footnotetext{
${ }^{1}$ Reactive power support is not considered at this stage.
}

As the cost function is convex and all constraints are linear, the optimization problem is convex. We also note that, even if the charging horizon is defined for a fixed time range, one can accommodate arbitrary arrival and departure times by enforcing zero charging power with new linear equality constraints. For example, a predicted departure time at $t=T-1$ for vehicle $v$ can be modeled by adding $P_{v t}^{(\mathrm{EV})}=0$ to the constraints.

\section{B. Scheduling the charge of autonomous EVs}

a) Intuition: Autonomous EVs can pick independently a charging station to accelerate their recharging process and diminishing the impact on the grid. As opposed to the previous problem, the binary variables $b_{n v}$ are no longer predetermined by the parking locations of the vehicles and are now part of the decision problem, which therefore becomes a mixed integer problem. As the charging locations are now determined by the optimization problem, we need to enforce consistency in the model and ensure that the each vehicle is at one location only. We refer to this requirement as the non-multilocation constraint and it reads as:

$$
\sum_{n=1}^{N} b_{n v} \leq 1
$$

b) Bi-linear relationships in the nodal injections: Eq. (2) requires special attention as it features products among decision variables, leading to a complex bi-linear formulation. We use the McCormick's relaxation [18] and replace the bi-linear constraint in (2)

$$
z_{n v t}=b_{n v} P_{t v}^{(\mathrm{EV})}
$$

where $b \in\{0,1\}$ and $0 \leq P_{n v t}^{(\mathrm{EV})} \leq \bar{P}_{v}^{(\mathrm{EV})}$, with the linear inequality constraints

$$
\begin{aligned}
& z_{n v t} \leq b_{n v} \bar{P}_{v}^{(\mathrm{EV})} \\
& z_{n v t} \leq P_{t v}^{(\mathrm{EV})} \\
& z_{n v t} \geq P_{t v}^{(\mathrm{EV})}-\bar{P}_{v}^{(\mathrm{EV})}\left(1-b_{n v}\right)
\end{aligned}
$$

As $b_{n v}$ are binary variables, the relaxation in (11) is exact.

c) Additional charge required for autonomous driving:

The round-trip drive between the drop-off and charging locations increases the required charging demand to some extent. The additional demand per lag is conservatively estimated by the maximum pairwise distance among grid nodes times the electric energy per unitary distance and is denoted $E^{*}$. As detailed in the following paragraph we will use the same modelling framework as (8), where the binary variables $b_{n v}$ are let free. To model the requirements of autonomous driving in such a framework, we need to implement the following modeling considerations:

1) For a vehicle to undertake the trip to the charger, its residual SOC at the parking location should be larger than $E^{*}$. If this condition is not met, the vehicle is forced to charge locally by enforcing the respective binary variable $b_{n v}$ to parking location $b_{n v}^{*}$. Otherwise, $E^{*}$ is subtracted from the SOC so that it can be compensated for during the charging process. 
2) The target state-of-charge $S O C_{v}^{*}$ is incremented by an amount proportional to $E^{*}$ (subject to not incurring in overcharging) so that the energy demand required by the return trip is also compensated for.

3) After, the scheduling problem is completed, $S O C_{v}^{*}$ is curtailed by an amount proportional to $E^{*}$ to model the energy spent in the return trip.

As shown in the following, these modeling considerations can be implemented with simple pre- and post-optimization heuristics without impacting on the tractability of the problem. More complex partial recharging schemes (e.g., the vehicle achieves a partial charge and drive to a more suitable site for achieving the final charging goal), as well as an improved approximation of the driving distance accounting for road itineraries, will be considered in future works.

d) Formulation: The binary variables $b_{n v}$, which denote the charging locations of the EVs and were input in the previous formulation, are now decision variables of the problem, leading to a mixed integer program. By using the (exact) relaxation discussed above, we reformulate the bilinear relationships of (2) into tractable linear constraints. The optimal charging schedules and charging locations $b_{n v}^{o}$ are given by

$$
\underset{\substack{P_{11}^{(\mathrm{EV})}, \ldots, P_{T V}^{(\mathrm{EV})} \in \mathbb{R}_{+} \\ b_{11}, \ldots, b_{n v} \in\{0,1\}}}{\arg \min }\left\{\sum_{t=0}^{T} \sum_{v=1}^{V}\left(\operatorname{SOC}_{t v}\left(P_{t v}^{\mathrm{EV}}\right)-\mathrm{SOC}_{v}^{*}\right)^{2}\right\}
$$

subject to the non-multilocation constraint in (9) for all $n$ and $v$, nodal injections and McCormick's exact relaxation for bilinear constraints for all $t, v$ and $n$

$$
\begin{aligned}
& P_{t n}\left(\boldsymbol{P}_{t}^{(\mathrm{EV})}, \boldsymbol{b}_{n}\right)=P_{t n}^{(\mathrm{net})}+\sum_{v=1}^{V} z_{n v t} \\
& z_{n v t} \leq b_{n v} \bar{P}_{v}^{(\mathrm{EV})} \\
& z_{n v t} \leq P_{t v}^{(\mathrm{EV})} \\
& z_{n v t} \geq P_{t v}^{(\mathrm{EV})}-\bar{P}_{v}^{(\mathrm{EV})}\left(1-b_{n v}\right) \\
& P_{v}^{(\mathrm{EV})} \leq \bar{P}_{v}^{(\mathrm{EV})},
\end{aligned}
$$

and, for all $t$ and $v$, SOCs' evolution as in (8b)-(8c), and load flow's and grid's constraints as in (8f)-(8k) (not reported here for brevity) with the difference that the latter group, depending on the nodal injections, are also a function of the binary decision variables. Additionally, conditions 1-3 introduced in Paragraph III-B0c are implemented by:

$$
\begin{gathered}
\text { if }\left(S O C_{0 v} \geq E^{*} / E_{v}\right): S O C_{0 v}=S O C_{0 v}-E^{*} / E_{v} \\
\text { else }: b_{n v}=b_{n v}^{*}, \quad \text { for all } v \text { and } n \\
\operatorname{SOC}_{v}^{*}=\min \left(\operatorname{SOC}_{v}^{*}+E^{*} / E_{v}, 1\right),
\end{gathered}
$$

with a note on $(12 \mathrm{~h})^{2}$. Once the problem is solved, we subtract from the final SOC the energy required for the return trip from

\footnotetext{
${ }^{2} \mathrm{Eq}$. (12h) is a (conservative) modeling approximation as the additional demand $E^{*}$ should be implemented only when the charging and parking locations are different. The refinement of this modeling aspect will be considered in future works.
}

the charging spot to the original parking location, if different:

$$
\text { if }\left(b_{n v}^{o}<>b_{n v}^{*}\right): S O C_{T v}=S O C_{T v}-E^{*} / E_{v},
$$

for all $v$ and $n$. Procedures (12g) and (12i) are respectively applied before and after solving the optimization problem based on input and output data and do not alter the problem's properties and tractability.

\section{RESULTS AND DISCUSSION}

\section{A. Case study and input parameters}

a) Grid: We consider the topology (Fig. 1) and cable characteristics of the CIGRE benchmark system for LV residential grids [19]. The nominal active power demand and power factor are reported in Table I, and the trajectory of the nodal demand at the node, used to model nodal injections, in Fig. 2. They are all according to specs of the CIGRE benchmark system. The number of vehicles per node, also in Table I, is set assuming 1.5 vehicles per household, with number of households per node approximated by the nodal nominal demand divided the contractual power for households (e.g., 6 kVA in France). We consider 16 A chargers (i.e., 3.7 $\mathrm{kW}$ at nomimal voltage). The voltage limits are set to $1 \mathrm{pu} \pm$ $8 \%$, while lines ampacities are according to CIGRE' specs.

TABLE I

NOMINAL DEMAND PER NODE AND DISTRIBUTION OF ELECTRIC VEHICLES (THE LAST COLUMN REFERS TO RESULTS)

\begin{tabular}{|c|c|c|c|c|}
\hline Node & $\begin{array}{c}\text { Nominal } \\
\text { demand } \\
\text { (kW) }\end{array}$ & $\begin{array}{c}\text { Power } \\
\text { factor }\end{array}$ & $\begin{array}{c}\text { Number of } \\
\text { parked EVs }\end{array}$ & $\begin{array}{c}\text { Number of charging } \\
\text { AEVs (from results) }\end{array}$ \\
\hline 1 & 200 & 0.95 & 50 & 54 \\
11 & 15 & 0.95 & 3 & 6 \\
15 & 52 & 0.95 & 12 & 5 \\
16 & 55 & 0.95 & 14 & 15 \\
17 & 35 & 0.95 & 8 & 10 \\
18 & 47 & 0.95 & 11 & 6 \\
\hline
\end{tabular}

b) Electric vehicles: As in [7], we sample the EVs' departing times and SOC at arrival from Weibull (scale 7.67, shape 21.83) and Gaussian (mean 0.49, standard deviation 0.04) distributions, respectively, as shown in Fig. 3. Statistics are estimated from measurements of the test-an-ev experiment in Denmark [20], [21]. For simplicity and to better quantify the impact of autonomous vs. non-autonomous driving, we assume that the arrival time is the same for all vehicles. We adopt the same energy capacity of the EVs used in the experiment, i.e. $16 \mathrm{kWh}$, so as to retain consistency among collected state-ofcharge statistics and driving energy demand.

c) Sampling time and scheduling horizon: We use a sampling time of 1 hour; based on the $\min / \max$ values of the arrival times distribution (approximated to the nearest integer hour in-line with the adopted time resolution), we set the scheduling horizon of the problem to be from $16 \mathrm{~h}$ to $8 \mathrm{~h}$ of the next day.

d) Input parameters related to charging objectives: The target state-of-charge implemented in the cost functions of problems (8) and (12) is $S O C_{v}^{*}=100 \%$ for all vehicles $v=1, \ldots, V$. The additional charge $E^{*}$ for accomplishing 


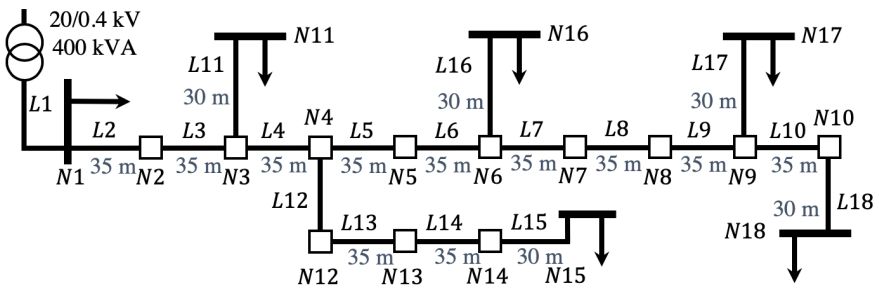

Fig. 1. Topology of European LV distribution network benchmark for residential system used for the verification [19].

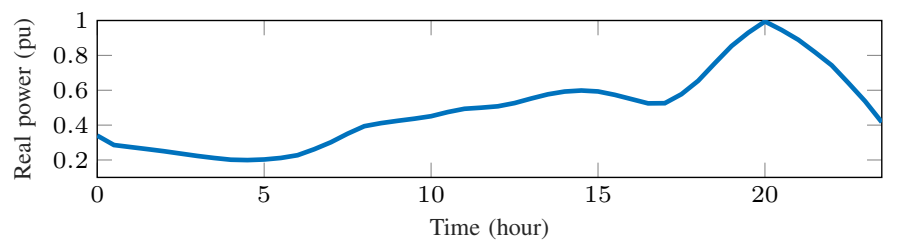

Fig. 2. Profile of the net active power demand from CIGRE specifications [19].

the autonomous drive to the charging station is the the maximum distance among nodes (345 meters, N18-N1 in Fig. 1) times the average consumption per $\mathrm{km}(0.160 \mathrm{kWh} / \mathrm{km}$ from experimental data [22]). It amounts to $55 \mathrm{Wh}$, i.e. $0.8 \%$ SOC.

\section{B. Metrics for performance evaluation}

Evaluating the cost function of the optimization problems offers an immediate interpretation of the performance of the scheduler along the charging horizon. Based on it, the first metric is:

$$
\text { Metric } 1=\sum_{t=1}^{T} \sum_{v=1}^{V}\left(S O C_{t v}-S O C_{t v}^{*}\right)^{2}
$$

The second metric evaluates the performance at the end of the scheduling period $T$ and measures if target charging objectives have been met:

$$
\text { Metric } 2=\sum_{v=1}^{V}\left(S O C_{T v}^{*}-S O C_{T v}\right) \text {. }
$$
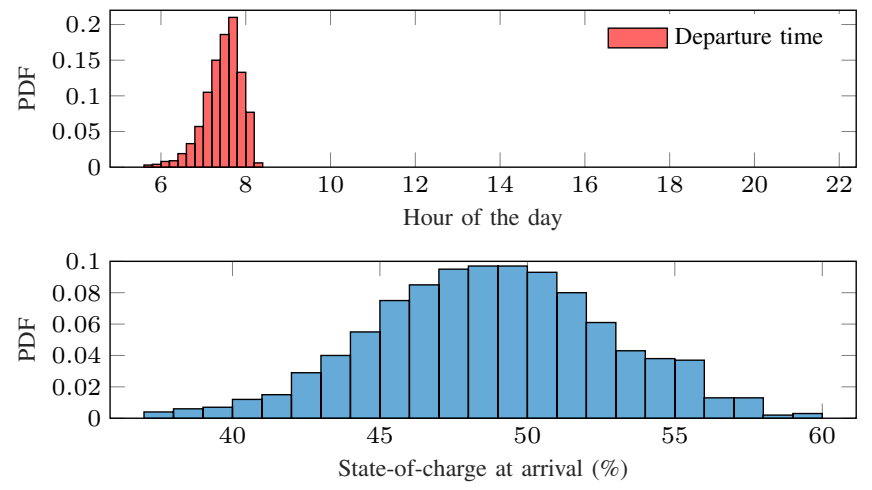

Fig. 3. Distributions of the EVs' departure times (upper-panel plot) and of the initial SOC (lower-panel plot) used in the simulations.
Finally, Metric 3 is the time taken by the charging process to reach the target SOC for all vehicles.

\section{Results}

The number of AEVs that are charged at the various nodes of the grid is shown in the last column of Table I. It can be seen that, compared to the case of non-autonomous EVs, 13 AEVs pick a different charging location. As to be expected, AEVs tend to favor charging locations closer to the GCP, that allow connecting higher demand with less impact on voltage levels. For example, nodes 1 and 11 feature additional 4 and 3 charging vehicles than parked vehicles, as opposed to remote nodes 15 and 18 that are with less vehicles.

The upper- and middle-panel plots of Fig. 4 show the charging power and SOCs' evolution at different quantiles of the population of EVs, respectively. AEVs feature a higher degree of simultaneity when charging and achieve the target SOC quicker than conventional EVs. Thanks to picking charging locations closer to the GCP, AEVs achieve simultaneous charging while respecting voltage constraints as visible in the middle-panel plot of Fig. 4.

Table II shows that autonomous EVs achieve a lower realization of the cost function (Metric 1) and the target SOC in 4 hours, nearly half of the time than conventional vehicles (Metric 3) ${ }^{3}$. Conventional EVs score better in Metric 2 because those autonomous vehicles which change locations for charging cannot achieve $100 \%$ SOC as some charge is spent in driving back to the original parking location.

TABLE II

METRICS FOR CONVENTIONAL VS. AUTONOMOUS VEHICLES.

\begin{tabular}{|c|c|c|}
\hline Metric & Conventional & Autonomous \\
\hline Metric 1 & 373 & 300 \\
Metric 2 & $0 \%$ & $0.8 \%$ \\
Metric 3 & 7 hour & 4 hour \\
\hline
\end{tabular}

\section{Conclusions}

We have considered the problem of scheduling the charging demand of autonomous EVs, embedding the notion that they can drive autonomously to a suitable charging location to avoid grid congestions. The scheduling problem is a mixedinteger program. The charging demand of the autonomous EVs was mapped to nodal injections with bilinear relationships among the decision variables, which were reformulated with a McCormick (exact) relaxation to attain a linear formulation. Grid constraints on nodal voltage magnitudes, line ampacities, and power flow at the grid connection point were formulated with a linearized grid model based on sensitivity coefficients.

By enforcing the charging locations of the EVs a priori, the same algorithmic framework can solve the charge scheduling problem for non-autonomous EVs. In this case, the optimization problem is convex. The schedulers' performance for nonautonomous and autonomous EVs was compared in terms of

\footnotetext{
${ }^{3}$ As the distance among nodes is small (i.e., $<350$ meters), we neglect the time it takes to drive from the charging location to the parking location
} 

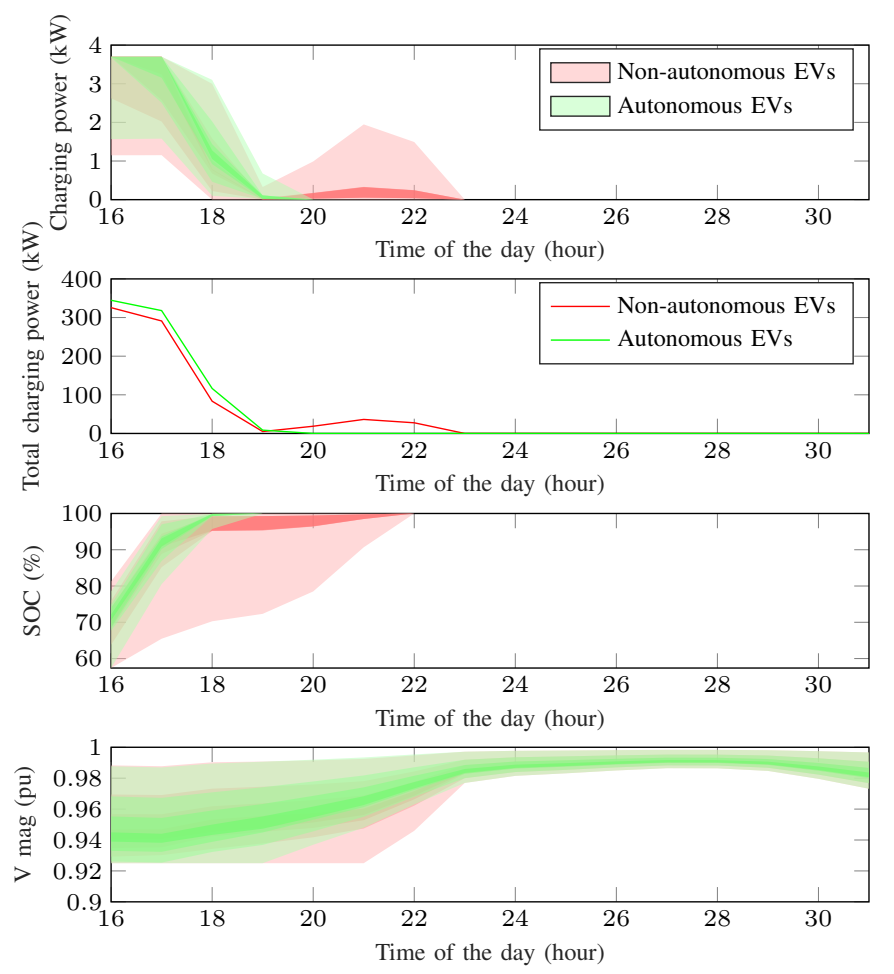

Fig. 4. Charging power (first panel), total charging power, and SOC (third panel) of the EVs population, and nodal voltage magnitude (fourth panel) of all grid nodes. In the $1 \mathrm{st}$, 3rd, and 4 th plots, the lighter color-shade refers to the 0 and 1 quantiles, the thicker to the 0.43 and 0.57 ones, whereas the two primary colors red and green refer to conventional and autonomous EVs, respectively.

how fast they can satisfy the charging demand while subject to all grid constraints. The performance comparison was carried out by considering the CIGRE' benchmark system for LV residential grids and realistic EVs' data. The rating of the grid is $400 \mathrm{kVA}$. We have considered a population of $96 \mathrm{EVs}$ charging with $3.6 \mathrm{~kW}$ chargers operating at a unitary power factor.

Simulation results show that AEVs can achieve the same charging demand in nearly half of the time than nonautonomous EVs. In the considered case study, the additional charging of autonomous EVs was a fraction (less than 1\%) of the EVs' battery energy capacity due to the small extension of the grid (the main feeder is less than $400 \mathrm{~m} \mathrm{long}$ ) and had a negligible impact on additional well-to-wheel emissions.

The future work is in the direction of extending the framework to unbalanced grids, including the effect of reactive power and evaluating the impact on road traffic.

\section{REFERENCES}

[1] H. Zhang, S. Moura, Z. Hu, W. Qi, and Y. Song, "Joint pev charging station and distributed pv generation planning," in 2017 IEEE power \& energy society general meeting. IEEE, 2017, pp. 1-5.

[2] Z. Lin, Z. Hu, and Y. Song, "Optimal planning of paired pev charging stations and esss in distribution networks considering flexibility of charging loads," in 2019 IEEE Power Energy Society General Meeting (PESGM), Aug 2019, pp. 1-5.
[3] IRENA, "Innovation outlook: Smart charging for electric vehicles," International Renewable Energy Agency, Abu Dhabi, Tech. Rep. MSUCSE-06-2, 2019.

[4] O. Sundström and C. Binding, "Planning electric-drive vehicle charging under constrained grid conditions," in 2010 International Conference on Power System Technology. IEEE, 2010, pp. 1-6.

[5] O. Sundstrom and C. Binding, "Flexible charging optimization for electric vehicles considering distribution grid constraints," IEEE Transactions on Smart Grid, vol. 3, no. 1, pp. 26-37, 2011.

[6] J. Hu, S. You, M. Lind, and J. Østergaard, "Coordinated charging of electric vehicles for congestion prevention in the distribution grid," IEEE Transactions on Smart Grid, vol. 5, no. 2, pp. 703-711, 2013.

[7] K. Knezović, A. Soroudi, A. Keane, and M. Marinelli, "Robust multiobjective pq scheduling for electric vehicles in flexible unbalanced distribution grids," IET Generation, Transmission \& Distribution, vol. 11, no. 16, pp. 4031-4040, 2017.

[8] A. Masood, J. Hu, A. Xin, A. R. Sayed, and G. Yang, "Transactive energy for aggregated electric vehicles to reduce system peak load considering network constraints," IEEE Access, vol. 8, pp. 31519 $31529,2020$.

[9] O. for Economic Co-operation and Development, Urban mobility system upgrade: How shared self-driving cars could change city traffic. OECD Publishing, 2015.

[10] R. Iacobucci, B. McLellan, and T. Tezuka, "Optimization of shared autonomous electric vehicles operations with charge scheduling and vehicle-to-grid," Transportation Research Part C: Emerging Technologies, vol. 100, pp. 34-52, 2019.

[11] M. Lokhandwala and H. Cai, "Siting charging stations for electric vehicle adoption in shared autonomous fleets," Transportation Research Part D: Transport and Environment, vol. 80, p. 102231, 2020.

[12] H. Zhang, C. J. Sheppard, T. E. Lipman, T. Zeng, and S. J. Moura, "Charging infrastructure demands of shared-use autonomous electric vehicles in urban areas," Transportation Research Part D: Transport and Environment, vol. 78, p. 102210, 2020.

[13] M. Kraning, Y. Wang, E. Akuiyibo, and S. Boyd, "Operation and configuration of a storage portfolio via convex optimization," in Proceedings of the 18th IFAC World Congress, vol. 18. Citeseer, 2011, pp. $10487-$ 10492.

[14] F. Sossan, E. Namor, R. Cherkaoui, and M. Paolone, "Achieving the dispatchability of distribution feeders through prosumers data driven forecasting and model predictive control of electrochemical storage," IEEE Transactions on Sustainable Energy, vol. 7, no. 4, pp. 1762-1777, Oct 2016.

[15] E. Stai, L. Reyes-Chamorro, F. Sossan, J. Y. L. Boudec, and M. Paolone, "Dispatching stochastic heterogeneous resources accounting for grid and battery losses," IEEE Transactions on Smart Grid, vol. 9, no. 6, pp. 6522-6539, Nov 2018.

[16] E. Stai, F. Sossan, E. Namor, J. Y. L. Boudec, and M. Paolone, "A receding horizon control approach for re-dispatching stochastic heterogeneous resources accounting for grid and battery losses," (to appear in) Elsevier Electric Power Systems Research (EPSR), 2020.

[17] K. Christakou, D.-C. Tomozei, J.-Y. Le Boudec, and M. Paolone, "Gecn: Primary voltage control for active distribution networks via real-time demand-response," IEEE Transactions on Smart Grid, vol. 4, no. 2, pp. 622-631, 2013.

[18] G. P. McCormick, "Computability of global solutions to factorable nonconvex programs: Part i-convex underestimating problems," Mathematical programming, vol. 10, no. 1, pp. 147-175, 1976.

[19] C. T. F. C6.04.02, "Benchmark systems for network integration of renewable and distributed energy resources,' Cigre' International Council on large electric systems, Tech. Rep., July 2009.

[20] P. B. Andersen, "Intelligent electric vehicle integration-domain interfaces and supporting informatics," Ph.D. dissertation, Technical University of Denmark (DTU), 2013.

[21] "Test-an-ev project: Electrical vehicle (ev) data," http://mclabprojects.di.uniroma1.it/smarthgnew/Test-an-EV/?EVcode $=$ EV8, accessed: 2020-02 .

[22] Clever A/S, "Test-en-elbil slutrapport," https://sonderborgkom.dk/wpcontent/uploads/Test-en-elbil-slutrapport-2014.pdf, Clever A/S, Tech. Rep., July 2014. 